\title{
Nanoroughness localization of excitons in GaAs multiple quantum wells studied by transient four-wave mixing
}

Birkedal, Dan; Vadim, Lyssenko; Pantke, Karl-Heinz; Østergaard, John Erland; Hvam, Jørn Märcher

Published in:

Physical Review B

Link to article, DOI:

10.1103/PhysRevB.51.7977

Publication date:

1995

Document Version

Publisher's PDF, also known as Version of record

Link back to DTU Orbit

Citation (APA):

Birkedal, D., Vadim, L., Pantke, K-H., Østergaard, J. E., \& Hvam, J. M. (1995). Nanoroughness localization of excitons in GaAs multiple quantum wells studied by transient four-wave mixing. Physical Review B, 51(12), 7977-7980. https://doi.org/10.1103/PhysRevB.51.7977

\section{General rights}

Copyright and moral rights for the publications made accessible in the public portal are retained by the authors and/or other copyright owners and it is a condition of accessing publications that users recognise and abide by the legal requirements associated with these rights.

- Users may download and print one copy of any publication from the public portal for the purpose of private study or research.

- You may not further distribute the material or use it for any profit-making activity or commercial gain

- You may freely distribute the URL identifying the publication in the public portal 


\title{
Nanoroughness localization of excitons in GaAs multiple quantum wells studied by transient four-wave mixing
}

\author{
D. Birkedal, V. G. Lyssenko, ${ }^{*}$ K.-H. Pantke, ${ }^{\dagger}$ J. Erland, ${ }^{\ddagger}$ and J. M. Hvam \\ Mikroelektronik Centret, The Technical University of Denmark, DK-2800 Lyngby, Denmark
}

(Received 16 January 1995)

\begin{abstract}
The interface roughness on a nanometer scale plays a decisive role in dephasing of excitons in GaAs multiple quantum wells. The excitonic four-wave mixing signal shows a free polarization decay and a corresponding homogeneously broadened line from areas with interface roughness on a scale larger than the exciton diameter. A photon echo and a corresponding inhomogeneously broadened line are observed from areas of interface roughness on a scale less than the exciton diameter. In the present study we observe both mechanisms simultaneously, and are able to clearly distinguish between the two mechanisms by spectrally resolving the transient four-wave-mixing signal.
\end{abstract}

The dephasing mechanisms of optical excitations in semiconductor quantum wells have recently attracted considerable attention. Ultrafast optical excitation results in coherent nonlinear effects such as free polarization decay, ${ }^{1}$ photon echoes, ${ }^{2}$ quantum beats, ${ }^{3,4}$ and other wave packet effects. ${ }^{5,6}$ These phenomena have been described extensively using either the optical Bloch equations or the semiconductor Bloch equations, the latter taking the microscopic description of the semiconductor into account. ${ }^{7,8}$

However, the description of nonlinear coherent phenomena in semiconductor heterostructures is further complicated due to imperfections of the semiconductor heterointerface. The formation of monolayer islands during molecular-beamepitaxial (MBE) growth is a well-known phenomena in the case of $\mathrm{GaAs} / \mathrm{Al}_{x} \mathrm{Ga}_{1-x} \mathrm{As}$. The island sizes, distribution, and their influence on the linear and nonlinear optical properties have been the object of several studies. ${ }^{9-14}$ The advances in MBE growth technology have improved sample quality. Earlier low temperature photoluminescence experiments suggested that excitonic absorption lines from multiple quantum wells (MQW's) were inhomogeneously broadened due to confinement fluctuations ${ }^{9}$ and that islands were large and atomically flat. Since then a number of investigations have addressed the problem ${ }^{10-14}$ resulting in the present model of the interface in which monolayer flat islands with a spatial extension of 50-100 $\AA$ are prevailing. The exciton diameter in $\mathrm{GaAs} / \mathrm{Al}_{x} \mathrm{Ga}_{1-x}$ As quantum wells is of the same magnitude as the monolayer islands size. Therefore, the electronic and optical properties are expected to be sensitive to whether the exciton diameter is smaller or larger than the island size, indicated by the existence of at least two length scales for the interface roughness. ${ }^{12,15}$

Several low temperature nonlinear optical measurements involving excitons in quantum well structures have addressed the problem. Transient four-wave-mixing (TFWM) experiments showed that the signal from MQW's is in the form of a photon echo, ${ }^{2}$ in accordance with the inhomogeneous broadening of the exciton resonance. ${ }^{16}$ Later experiments have shown homogeneously broadened lines ${ }^{1}$ and recently published results have shown cases where no clear distinction on the type of broadening is apparent, depending on either experimental condition or combination of model parameters. $^{17-20}$
In this paper we are able to simultaneously distinguish clearly between either predominantly homogeneously or inhomogeneously broadened parts of the TFWM signal by a spectral analysis. We are able to describe the TFWM signal as a combination of free polarization decays and photon echoes. The TFWM signal from the MQW sample is shown to be composed of a homogeneously broadened signal from atomically smooth areas of sizes larger than the exciton diameter and an inhomogeneously broadened signal from regions of roughness on a scale less than the exciton diameter.

We have investigated a MQW structure grown by MBE on a (001) semi-insulating substrate. The sample consisted of 20 wells of $100-\AA \mathrm{GaAs}$ separated by $200-\AA \mathrm{Al}_{0.3} \mathrm{Ga}_{0.7} \mathrm{As}$ barriers. The structure was lifted off the substrate using a selective etch technique and mounted on a sapphire disk. Photoluminescence measurements showed that mounting introduced only negligible homogeneous strain. Transmission measurements of the sample showed a heavy hole exciton absorption peak at $1.5500 \mathrm{eV}$ with a full width of $1.4 \mathrm{meV}$. The light hole exciton showed an absorption peak at 1.5580 $\mathrm{eV}$ with a width of approximately $2 \mathrm{meV}$. The Stokes shift of the heavy hole exciton peak was approximately $0.5 \mathrm{meV}$.

During the TFWM experiments the sample was mounted in a $\mathrm{He}$ cryostat held at $5 \mathrm{~K}$. The sample was excited by 150 -fs transform-limited pulses from a self-mode locked Ti:sapphire laser. The laser spot was focused to approximately $40 \mu \mathrm{m}$ at the sample surface. The TFWM experiment was performed in the two pulse self-diffracted geometry. In this geometry, two pulses with wave vectors $\boldsymbol{k}_{1}$ and $\boldsymbol{k}_{2}$ are incident on the sample under a small angle. The pulse with $\boldsymbol{k}_{2}$ arrives with a delay of $\tau$ after the first pulse with $\boldsymbol{k}_{1}$. The signal is then diffracted in the direction of $2 \boldsymbol{k}_{2}-\boldsymbol{k}_{1}$. The TFWM signal was dispersed in a spectrometer and detected by a cooled optical multichannel analyzer with a resulting resolution better than $0.1 \mathrm{meV}$. Due to the slow response of the detector we record the time integrated TFWM signals as a function of delay.

The spectrally resolved TFWM signal as a function of time delay is shown in Fig. 1(a) seen from negative delay and in Fig. 1(b) from positive delays. The spectrum is showing a doublet structure at near zero delay, decaying into a single line for larger time delays. The splitting of the heavy 

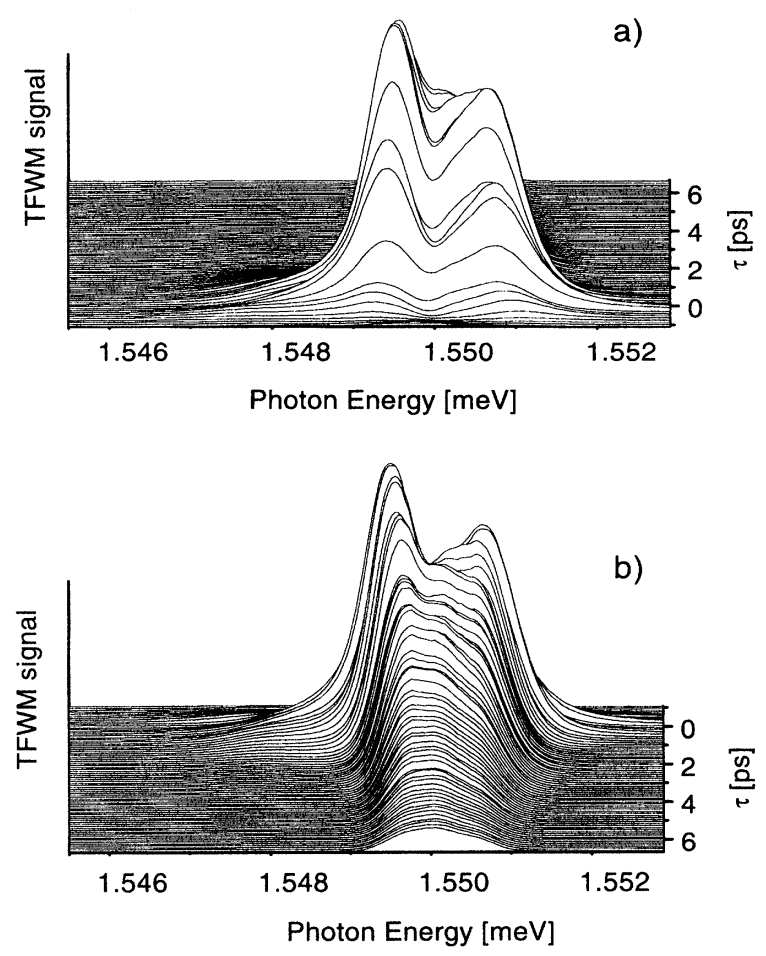

FIG. 1. Spectrally resolved TFWM signal seen from negative delay (a) showing the splitting near zero delay and from positive delay (b) showing the single line for long delays.

hole exciton peak is $1.35 \mathrm{meV}$ whereas the splitting of the light hole exciton peak is $1.40 \mathrm{meV}$ (outside of the spectral range of the figure). For longer delays ( $\tau>2 \mathrm{ps}$ ) the signal develops into a singlet structure centered near the average value of the two initial peaks.

This remarkable evolution of the TFWM signal is explained in the following way: The TFWM signal is composed of contributions from regions of different morphology. The two peaks near $\tau=0$ are free polarization decay originating from extended atomically smooth areas larger than the exciton diameter with a difference of one atomic layer in thickness. The splitting of the peaks of $1.35 \mathrm{meV}$ corresponds to the exciton energy shift of one atomic step of a 100- $\AA$-wide well. The single peak observed for longer delays is a time integrated photon echo from regions of the sample with roughness on a length scale smaller than the exciton diameter (nanoroughness). The different types of regions are schematically shown in Fig. 2. In Fig. 2(a) are shown two smooth areas I and III with a difference of one monolayer in thickness, and an area II where the thickness fluctuates between the thicknesses of regions I and III. Figure 2(b) shows the corresponding electron and exciton potentials, denoted by $E_{c}$ and $E_{x}$, respectively. The effect of nanoroughness is seen in region II, where the averaging effect is caused by the lateral extension of the exciton $a_{0}$. In the regions of nanoroughness the resulting exciton energy is distributed between the values of the two smooth areas. Due to the statistical nature of the growth, these values are expected to be normally distributed with a mean value near the average energy of the two smooth areas.
I II III

AlGaAs
GaAs

a)

\section{AIGaAs}

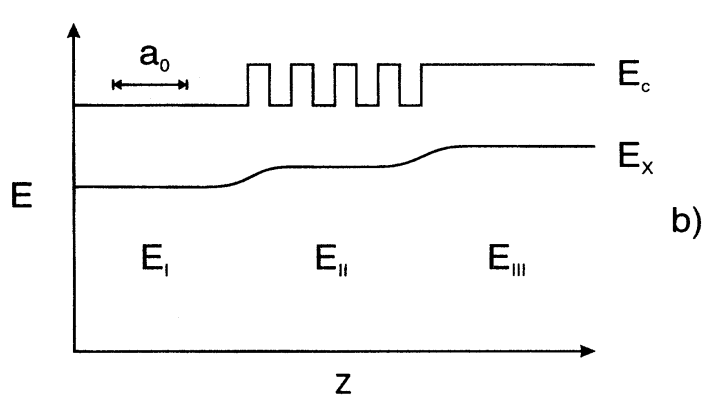

FIG. 2. Schematic representation of different types of interfaces (a), consisting of well regions of large smooth islands with a difference in thickness of one atomic layer as seen in regions I and III and a region II of roughness on a scale smaller than the exciton diameter $a_{0}$. Also shown in (b) are the electron and exciton energies, denoted by $E_{c}$ and $E_{x}$, respectively, for the structure shown in (a).

The theoretical TFWM line shapes for homogeneously and inhomogeneously broadened two-level systems have been investigated earlier, ${ }^{16,8}$ and recently by Erland et al. ${ }^{21}$ where spectral resolution of the TFWM signal was considered. These works are based on a perturbation expansion of the density matrix for a two-level system in the selfdiffracted geometry. ${ }^{16}$ The main result for the homogeneously broadened system is that the TFWM line shape is a Lorentzian with a half width of $1 / T_{2}$ where $T_{2}$ is the dephasing time of the two-level system, and the intensity of the line decays as a function of delay with a decay time of $T_{2} / 2$. Including local field effects, Wegener et al. ${ }^{8}$ found that signals for negative delay were present, growing as a function of delay with a rise time of $T_{2} / 4$. For the inhomogeneously broadened system the signal is emitted as a photon echo and for long delays the signal decays as a function of delay with a decay time of $T_{2} / 4$. Furthermore, the maximum of the signal is found for a finite positive delay and including local field effects no signal was found for negative delay. ${ }^{8}$ For long delays the line shape was found to be Gaussian with a delay independent width. ${ }^{21}$

On the basis of these findings we conclude that the two peaks near $\tau=0$ are due to free polarization decay of excitons created in regions of smooth and atomically flat interfaces of sizes larger than the exciton diameter. Two Lorentzian line shapes can easily be fitted to the spectrum of the TFWM signal for a small negative delay $(\tau=-0.5 \mathrm{ps})$ as shown in Fig. 3. The full width of these fitted Lorentzians are 


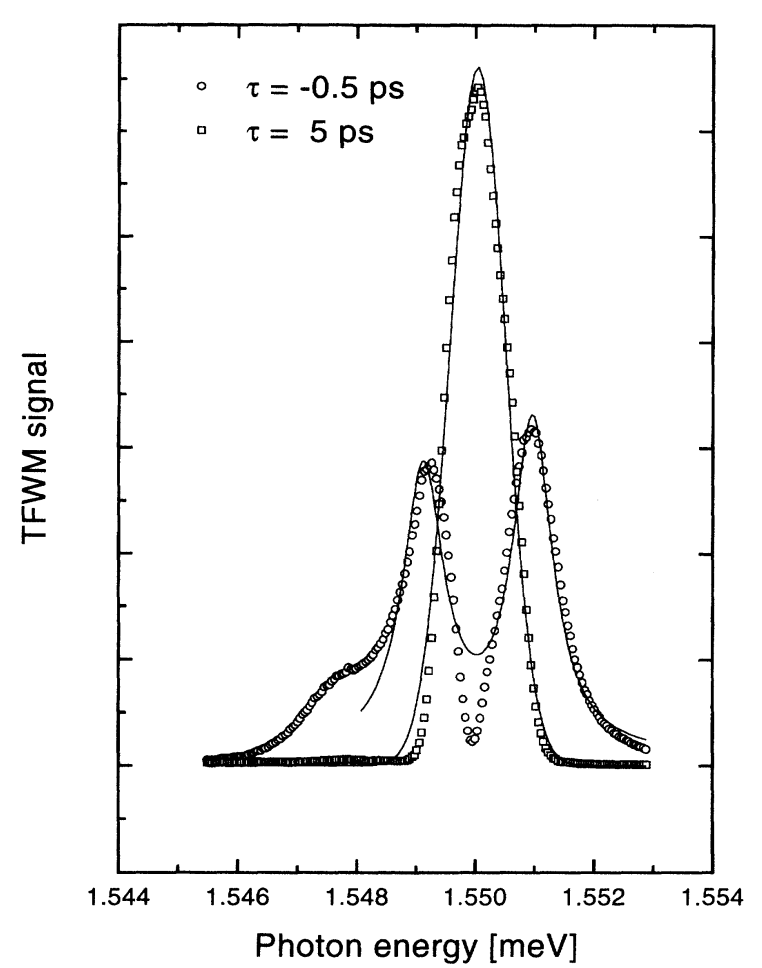

FIG. 3. Spectral shape of the TFWM signal (circles and squares) and Lorentzian and Gaussian line shape fits (solid lines) at -0.5 -ps and 5-ps delays, respectively.

approximately $1 \mathrm{meV}$ corresponding to a $T_{2}$ of $1.3 \mathrm{ps}$. It is difficult to evaluate the decay rate of the free polarization decay for positive delay as it is mixing with the single photon echo line appearing for positive delays. However, we find an exponential growth of the signal for negative delays at a rate of $0.25 \mathrm{ps}$, indicating a dephasing time of approximately 1 ps in reasonable agreement of that deduced from the Lorentzian fits.

For longer delay times we find that the line shape is Gaussian as shown in Fig. 3, reflecting the inhomogeneous broadening of the system. The delayed maximum of the central spectral component is also a strong indication of an inhomogeneously broadened system, as discussed previously. Hence, the signal for long delays originates from a different region of the sample than the signal for small delays since this signal was shown to be homogeneously broadened. The center of the line has a characteristic decay time of 2.4 ps indicating a $T_{2}$ of $9.4 \mathrm{ps}$. The large difference between the dephasing times deduced from the homogeneous and the inhomogeneous parts of the TFWM signal reflects that localization of excitons leads to an increase of the dephasing time. ${ }^{22,19}$ The excitons created in the regions of nanoroughness are localized and are less likely to undergo scattering events. However, excitons created in the regions of large and smooth islands are extended states and are more likely to undergo scattering events (exciton-exciton and excitonimpurity) resulting in enhanced dephasing. ${ }^{1}$

The Gaussian line for long delays is centered in the middle of the two initial peaks and the width is less than the initial splitting, supporting the aforementioned interface

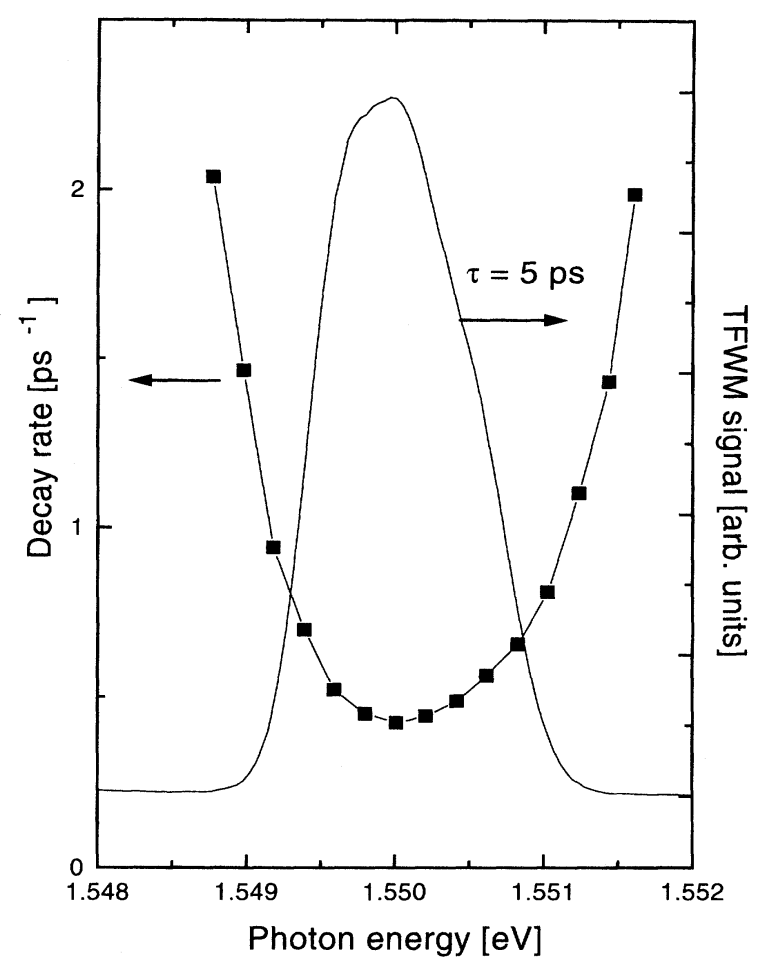

FIG. 4. The decay rate of the TFWM signal measured at different spectral positions for long delays. Also shown is the transient TFWM line shape at a delay of 5 ps.

model. However, such a model implies localization of the excitons at different local potential minima. To investigate the effects of localization further we have plotted the decay rate of the TFWM signal for long decay times as a function of energy in Fig. 4 together with the spectrum of the FWM signal for a delay of 5 ps. The remarkable result shows a clear minimum of the decay rate at the center of the line. A lower decay rate usually implies a stronger localization. ${ }^{22}$ Hence, the central part of the signal represents states of stronger localization than those in the wings of the signal. This is well explained within the present interface model. The states in the wings of the signal are created in regions of islands with similar or slightly larger sizes as the exciton diameters. These states are more weakly localized and approach the characteristics of the delocalized excitons, with a faster dephasing rate.

In the classical picture of Anderson localization ${ }^{23,24}$ the lowest energy state is most strongly localized. In addition, the localized and the delocalized states cannot overlap in energy. A localized state would always be able to tunnel out into the delocalized state degenerate with it. Consequently, there must be a mobility edge separating the localized states with the delocalized states. ${ }^{25}$ At first sight, our TFWM result seems to contradict these statements, having a delocalized state with lower energy than the localized. However, the classical picture of localization governs the quasiequilibrium state of the system. The coherent TFWM experiment samples the nonequilibrium state of the excited system and any tunneling will cause a loss of coherence. Eventually, all the excitons will scatter to the lowest lying islands where they 
recombine, as evidenced in our photoluminescence measurements. The nanoroughness localization is therefore only seen directly in the coherent experiment, where only the initially created coherent excitons contribute to the signal. However, nanoroughness is also expected to influence noncoherent properties, e.g., transport, as well. ${ }^{26}$

In conclusion, we have shown that using spectrally resolved TFWM we are able to extract information about the microscopic nature of the dephasing mechanisms of excitons in quantum wells. We have demonstrated that excitons local- ized on nanoroughness potential fluctuations do not localize as in the classical Anderson picture. The effects of nanoroughness localization are only directly accessible in the coherent experiment, as the localized excitons will relax to the lower-lying delocalized states, losing their coherence.

We would like to thank C. B. Sørensen for the sample preparation. This work was supported by the Danish Natural Science Research Council.
* Permanent address: Institute of Microelectronics Technology and Superpure Materials, Chernogolovka, Moscow District, 142432, Russia.

${ }^{\dagger}$ Present address: Institut für Angewandte Photophysik, Technische Universität Dresden, 101089 Dresden, Germany.

${ }^{\ddagger}$ Present address: Fysisk Institut, Odense Universitet, DK-5230 Odense M, Denmark.

${ }^{1}$ L. Schultheis et al., Phys. Rev. B 34, 9027 (1986).

${ }^{2}$ L. Schultheis, M. Sturge, and J. Hegarty, Appl. Phys. Lett. 47, 995 (1985).

${ }^{3}$ K. Leo, T. Damen, J. Shah, and K. Köhler, Phys. Rev. B 42, 11359 (1990).

${ }^{4}$ K.-H. Pantke and J. M. Hvam, Int. J. Mod. Phys. B 8, 73 (1994).

${ }^{5}$ K. Leo et al., Phys. Rev. Lett. 66, 201 (1991).

${ }^{6}$ J. Feldmann et al., Phys. Rev. Lett. 70, 3027 (1993).

${ }^{7}$ M. Lindberg and S. Koch, Phys. Rev. B 38, 3342 (1988).

${ }^{8}$ M. Wegener, D. Chemla, S. Schmitt-Rink, and W. Schäfer, Phys. Rev. A 42, 5675 (1990).

${ }^{9}$ C. Weisbuch, R. Dingle, A. Gossard, and W. Wiegmann, Solid State Commun. 38, 709 (1981).

${ }^{10}$ D. Reynolds et al., Appl. Phys. Lett. 46, 51 (1985).
${ }^{11}$ M. Kohl et al., Phys. Rev. B 39, 7736 (1989).

${ }^{12}$ C. Warwick, W. Jan, A. Ourmazd, and T. Harris, Appl. Phys. Lett. 56, 2666 (1990).

${ }^{13}$ R. Kopf, E. Schubert, T. Harris, and R. Becker, Appl. Phys. Lett. 58, 631 (1991).

${ }^{14}$ C. Warwick and R. Kopf, Appl. Phys. Lett. 60, 386 (1992).

${ }^{15}$ D. Gammon, B. Shanabrook, and D. Katzer, Phys. Rev. Lett. 67, 1547 (1991).

${ }^{16}$ T. Yajima and Y. Taira, J. Phys. Soc. Jpn. 47, 1620 (1979).

${ }^{17}$ M. Webb, S. Cundiff, and D. Steel, Phys. Rev. B 43, 12658 (1991).

${ }^{18}$ T. Wang et al., J. Cryst. Growth 109, 285 (1991).

${ }^{19}$ S. Cundiff, H. Wang, and D. Steel, Phys. Rev. B 46, 7248 (1992).

${ }^{20}$ S. T. Cundiff, A. Knorr, J. Feldmann, S. W. Koch, E. O. Göbel, and H. Nickel, Phys. Rev. Lett. 73, 1178 (1994).

${ }^{21}$ J. Erland et al., Phys. Rev. B 50, 15047 (1994).

${ }^{22}$ J. Hegarty and M. Sturge, J. Opt. Soc. Am. B 2, 1143 (1985).

${ }^{23}$ P. Anderson, Phys. Rev. 109, 1492 (1958).

${ }^{24}$ P. Anderson, Physica 117/118B, 30 (1983).

${ }^{25}$ N. Mott, Philos. Mag. 13, 989 (1966).

${ }^{26}$ D. Oberhauser et al., Phys. Rev. B 47, 6827 (1993). 